\title{
Spin lattices of walking droplets
}

\author{
Pedro J. Sáenz, Giuseppe Pucci, Alexis Goujon, Tudor Cristea-Platon, \\ Jörn Dunkel, and John W. M. Bush \\ Department of Mathematics, Massachusetts Institute of Technology, \\ 77 Massachusetts Avenue, Cambridge, Massachusetts 02139, USA
}

(Received 4 February 2018; published 17 October 2018)

\begin{abstract}
This paper is associated with a video winner of a 2017 APS/DFD Gallery of Fluid Motion Award for work presented at the DFD Gallery of Fluid Motion. The original video is available from the Gallery of Fluid Motion, https://doi.org/10.1103/APS.DFD.2017.GFM.V0018
\end{abstract}

DOI: 10.1103/PhysRevFluids.3.100508

Millimetric liquid droplets may self-propel on the surface of a vibrating fluid bath through a resonant interaction with their own wave fields [1]. Such walking droplets, or walkers, have attracted considerable interest recently due to their capability to exhibit complex wave-particle behaviors previously thought exclusive to the microscopic quantum realm [2]. Couder and co-workers have explored the dynamics of walking droplets in the central force field resulting from a harmonic potential $[3,4]$. Their investigations revealed the emergence of quantized orbital states and a double quantization in the mean energy and angular momentum. In particular, the presence of a central force was shown to be essential for the realization of stable orbital states in the range of accessible experimental parameters [5-7]. Motivated by the wavelike statistics of a walking droplet in a circular corral [8], a recent study has demonstrated resonance phenomena in elliptical cavities [9], including a statistical projection effect reminiscent of the so-called quantum mirage [10]. The results in Ref. [9] further showed that pilot-wave dynamics is viable in relatively shallow water and that variations in bottom topography of the bath can be used to generate spatial gradients in the walker's memory, thereby effectively allowing for topographically induced potentials. Building on these insights, we consider here the hydrodynamic interactions of spinning walkers stabilized by bottom topography.

In our experiments, we confine walking droplets to circular wells surrounded by a relatively shallow liquid layer of depth $h$ [Fig. 1(a)]. When this layer is sufficiently thin $(h \ll 1 \mathrm{~mm})$ and the vibrational forcing sufficiently weak, both the droplet and its guiding wave remain confined to their underlying well [Fig. 1(b)]. For suitably chosen experimental parameters, however, the waves can extend substantially beyond the well boundaries even though the circular droplet trajectories remain confined to the well [Fig. 1(c)]. In this regime, the fluid layer between neighboring wells supports a wave-mediated interaction between adjacent spinning droplets, which may induce spin reversals when the pair coupling is sufficiently strong. To explore whether such wave-mediated spin-spin interactions may induce coherent collective dynamics, we first investigate the motion of spinning droplets on a submerged rectangular lattice [Figs. 2(a) and 2(b)]. Figure 2(c) illustrates the intricate instantaneous wave field that couples neighboring droplets. The emergence of collective behavior can be characterized through the mean spin-spin correlation $\chi=\left\langle\sum_{i \sim j} s_{i}(t) s_{j}(t)\right\rangle / N$, where $\sum_{i \sim j}$ denotes a sum over the $N$ adjacent spin pairs $\{i, j\}$ in the lattice and $\langle\cdot\rangle$ is a time average.

Published by the American Physical Society under the terms of the Creative Commons Attribution 4.0 International license. Further distribution of this work must maintain attribution to the author(s) and the published article's title, journal citation, and DOI. 
(a)

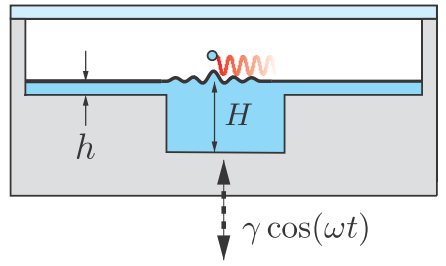

(b)

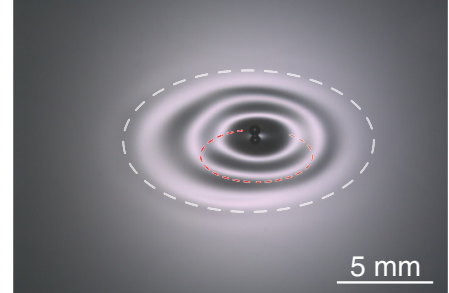

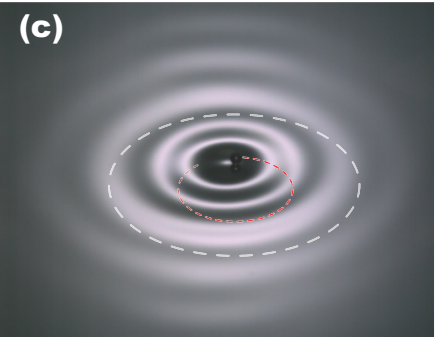

FIG. 1. A circular well at the bottom of a vibrated fluid bath forces a droplet to walk on a circular trajectory, and so 'spin' within the well. (a) Schematic of experimental setup. By tuning the depth of the surrounding liquid layer $h$, the range of the drop's guiding wave may either (b) be limited to the well domain ( $h \ll 1 \mathrm{~mm})$ or (c) extend beyond the well's edge $(h=1 \mathrm{~mm})$. Well boundaries are indicated by white dashed lines and particle trajectories in red.

Our preliminary results for square lattices indicate a preference for antiferromagnetic ordering, corresponding to $\chi<0$. A systematic investigation of the collective dynamics for different forcing parameters and lattice geometries (Fig. 3) is beyond the scope of the present work.
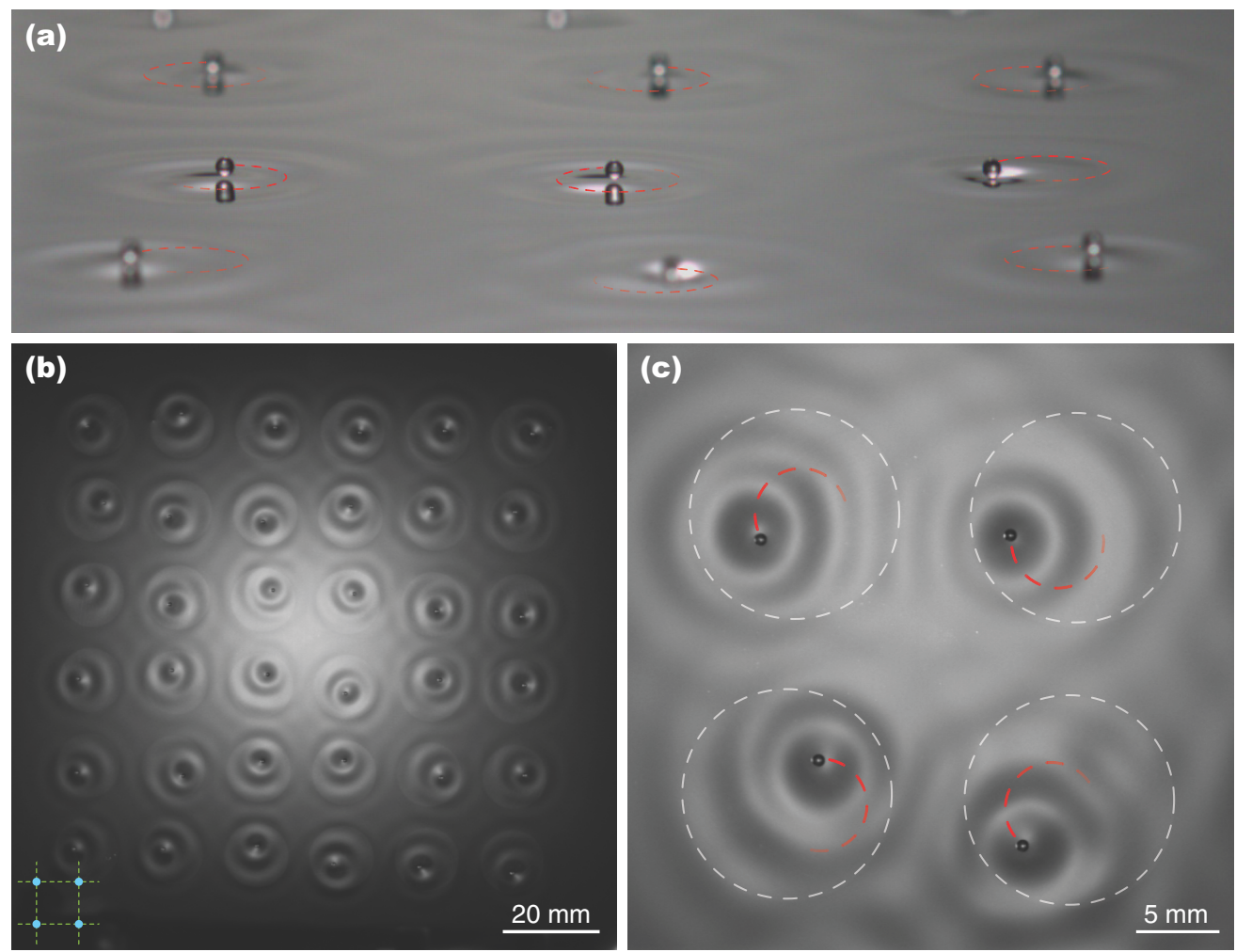

FIG. 2. Periodically distributed submerged wells can be used to construct stable spin lattices of walking droplets. The droplet dynamics are coupled through wave-mediated interactions between neighbors. (a) Oblique and (b) Plan views of a square lattice of spinning droplets. (c) Plan view of four neighboring droplets reveal the intricate instantaneous wave field resulting from their interactions. Well boundaries are indicated by white dashed lines and particle trajectories in red. 

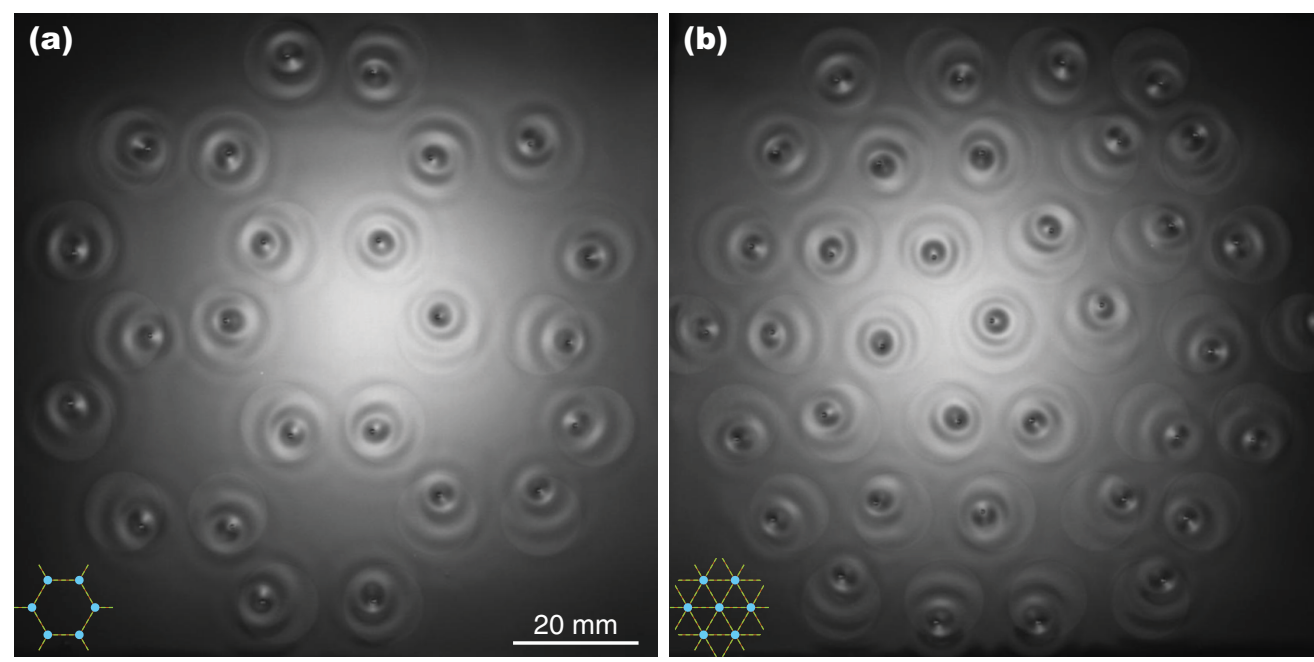

FIG. 3. Different forms of global order can emerge for non-square-lattice geometries, such as (a) hexagonal and (b) triangular spin lattices.

This work was supported by the US National Science Foundation through Grants No. CMMI-1333242, No. DMS-1614043, and No. CMMI-1727565.

[1] Y. Couder, S. Protiere, E. Fort, and A. Boudaoud, Dynamical phenomena: Walking and orbiting droplets, Nature (London) 437, 208 (2005).

[2] J. W. M. Bush, Pilot-wave hydrodynamics, Annu. Rev. Fluid Mech. 47, 269 (2015).

[3] S. Perrard, M. Labousse, M. Miskin, E. Fort, and Y. Couder, Self-organization into quantized eigenstates of a classical wave-driven particle, Nat. Commun. 5, 3219 (2014).

[4] S. Perrard, M. Labousse, E. Fort, and Y. Couder, Chaos Driven by Interfering Memory, Phys. Rev. Lett. 113, 104101 (2014).

[5] M. Labousse, S. Perrard, Y. Couder, and E. Fort, Self-attraction into spinning eigenstates of a mobile wave source by its emission back-reaction, Phys. Rev. E 94, 042224 (2016).

[6] E. Fort, A. Eddi, A. Boudaoud, J. Moukhtar, and Y. Couder, Path-memory induced quantization of classical orbits, Proc. Natl. Acad. Sci. USA 107, 17515 (2010).

[7] A. U. Oza, D. M. Harris, R. R. Rosales, and J. W. M. Bush, Pilot-wave dynamics in a rotating frame: On the emergence of orbital quantization, J. Fluid Mech. 744, 404 (2014).

[8] D. M. Harris, J. Moukhtar, E. Fort, Y. Couder, and J. W. M. Bush, Wavelike statistics from pilot-wave dynamics in a circular corral, Phys. Rev. E 88, 011001 (2013).

[9] P. J. Sáenz, T. Cristea-Platon, and J. W. M. Bush, Statistical projection effects in a hydrodynamic pilot-wave system, Nat. Phys. (2017), doi:10.1038/s41567-017-0003-x.

[10] G. A. Fiete and E. J. Heller, Theory of quantum corrals and quantum mirages, Rev. Mod. Phys. 75, 933 (2002). 